\title{
Spent Pleurotus ostreatus Substrate Has Potential for Managing Fusarium Wilt of Banana
}

\author{
Walter Ocimati $^{1, * \mathbb{D}}$, Evans Were ${ }^{2} \mathbb{D}$, Anthony Fredrick Tazuba ${ }^{1}$, Miguel Dita ${ }^{3} \mathbb{D}$, Si-Jun Zheng ${ }^{4} \mathbb{D}$ \\ and Guy Blomme ${ }^{5}$ (D)
}

check for updates

Citation: Ocimati, W.; Were, E.; Tazuba, A.F.; Dita, M.; Zheng, S.-J.; Blomme, G. Spent Pleurotus ostreatus Substrate Has Potential for Managing Fusarium Wilt of Banana. J. Fungi 2021, 7, 946. https://doi.org/ 10.3390/jof7110946

Academic Editor: Jeffrey J. Coleman

Received: 16 October 2021

Accepted: 5 November 2021

Published: 9 November 2021

Publisher's Note: MDPI stays neutral with regard to jurisdictional claims in published maps and institutional affiliations.

Copyright: (c) 2021 by the authors. Licensee MDPI, Basel, Switzerland. This article is an open access article distributed under the terms and conditions of the Creative Commons Attribution (CC BY) license (https:/ / creativecommons.org/licenses/by/ $4.0 /)$.
Alliance of Bioversity and CIAT, Kampala P.O. Box 24384, Uganda; tazubatony@gmail.com

2 Institute of Agricultural Sciences in the Tropics, University of Hohenheim, 70599 Stuttgart, Germany; e.were@uni-hohenheim.de

Alliance of Bioversity and CIAT, Cali 763537, Colombia; m.dita@cgiar.org

4 Alliance of Bioversity and CIAT, Kunming 650205, China; s.zheng@cgiar.org

5 Alliance of Bioversity and CIAT, Addis Ababa P.O. Box 5689, Ethiopia; g.blomme@cgiar.org

* Correspondence: w.ocimati@cgiar.org

\begin{abstract}
A range of basidiomycetes including the edible mushroom Pleurotus ostreatus (Po) can suppress plant pathogens such as Fusarium spp. With the current increase in production and consumption of Po in Uganda, the spent Po substrate (SPoS) could be an alternative to manage Fusarium wilt of banana (FWB), caused by the soil borne pathogen Fusarium oxysporum $\mathrm{f}$. sp. cubense, race $1(F o c)$. This study determined the potential of SPoS to inhibit Foc in vitro and in potted plants. In vitro studies confirmed suppression of Foc in pure co-culture (Po vs. Foc) assays and media amended with different concentrations $(0 \%$ to $50 \% w / v)$ of un-sterilized SPoS filtrates. Foc growth in the sterile SPoS filtrate was comparable to the water control, suggesting possible roles of biotic or thermolabile components of the SPoS. To further verify the suppressive effects of SPoS, pot experiments were carried out with a resistant ('Mbwazirume', AAA) and susceptible ('Sukali Ndizi', AAB) banana cultivar using both artificially and naturally infested soils. Independent of the inoculation method, SPoS significantly reduced the severity of FWB in pot experiments. Susceptible cultivar 'Sukali Ndizi' growing in substrates amended with SPoS showed lower (1.25) corm damage (Scale 0-5) than the un-amended control (3.75). No corm damage was observed in uninoculated controls. The resistant cultivar 'Mbwazirume', showed slight (0.25) corm damage only in the Focinoculated plants without SPoS. These findings suggest that SPoS could be used as part of the management practices to reduce the impact of FWB.
\end{abstract}

Keywords: basidiomycetes; biocontrol; corm damage; Fusarium oxysporum f. sp. cubense; metabolites; spent mushroom substrates

\section{Introduction}

The white rot fungus Pleurotus ostreatus is the second most important cultivated edible mushroom after Agaricus bisporus (white button mushroom) [1]. This fungus naturally lives as a saprophyte on dead or decaying wood and is cultivated as an edible mushroom using lignocellulosic wastes and agricultural by-products such as coffee husks, corn cobs and cotton seeds [2,3]. For every kilogram of mushroom, around $5 \mathrm{~kg}$ spent mushroom substrate (SMS) is produced [4]. After harvesting the fruiting bodies, the SMS is mostly discarded.

Edible mushrooms including $P$. ostreatus have been reported to play multiple roles in regenerative farming. The spent mushroom substrate (SMS) or spent mushroom compost (SMC) have a high organic matter content (22-40\%), high nutrient levels, high cation exchange capacity and slow mineralization, making these substrates efficient for plant growth [5,6]. Indeed, mushroom hyphae have been reported to wrap around plant roots and increase water and mineral availability for plants, thus improving plant yields. In maize (Zea mays), a 20\% yield increase has been reported when grown together with 
Stropharia rugoso-annulata, and in Brussels sprout (Brassica oleracea var. gemmifera), a 25\% yield increase has been reported when grown with Hypsizygus ulmarius [5,6].

The potential use of mushrooms as biocontrol agents of plant pathogens and crop pests has attracted considerable attention during the past few decades [7,8]. Mushrooms in the form of mushroom isolated compounds, the SMS, SMS extracts and SMC have been shown to affect plant pathogens. In the presence of abiotic or biotic stresses, such as interspecific interactions with other fast-growing fungi, $P$. ostreatus produces several secondary metabolites which have a wide range of physiological functions, including defense against invading microorganisms and survival under stressful conditions $[9,10]$. Mushroom extracts and isolated compounds, such as peptides and proteins, sesquiterpenes and other terpenes, steroids, organic acids and quinoline, have been shown to have antimicrobial activity [11]. During interspecific interactions with other fungi, the laccase activity of $P$. ostreatus has been associated with suppression of the growth of aerial mycelium, inducing severe cytological disturbances in several pathogenic fungi [12].

The SMS/SMC is composed of fungal mycelia, extracellular hydrolytic and oxidative enzymes secreted from mushrooms for the degradation of substrates, and unused, but partially modified, lignocellulosic substrates [13]. Spent mushroom compost tea was reported to have positive effects in the control of Phytophthora capsici and Phytophthora parasitica on pepper (Capsicum annuum L.) both in in vitro and in vivo assays [14]. The suppressive/antifungal effects of aqueous extracts of spent $P$. ostreatus substrate (SPoS) have also been reported against Fusarium oxysporum f. sp. lycopersici (Fol) in in vitro and in vivo studies [15]. The SPoS was also associated with diverse beneficial microorganisms including fluorescent Pseudomonas spp., Trichoderma viridae, Baccilus spp., Penicillum spp., and Aspergillus terrus, which showed strong antagonism to Fol [15].

The reports mentioned above suggest a suppressive effect of certain metabolites and microorganisms in the SPoS towards some plant pathogens. Suppression of Fol by SPoS also suggests that SPoS could potentially be used to manage Fusarium oxysporum $\mathrm{f}$. sp. cubense (Foc), the causal agent of Fusarium wilt of banana (FWB) [15]. FWB has severely impacted banana production worldwide and is currently considered as one of the major threats of this crop [16-18]. In Uganda, Foc race 1 has hampered the production of dessert banana cultivars (e.g., 'Gros Michel', Musa AAA and 'Sukali Ndizi' (syn. 'Ndizi'), Musa $\mathrm{AAB}$ ) and the $\mathrm{ABB}$ beer types (e.g., 'Pisang Awak' and 'Bluggoe').

In infested soils, Foc can survive for decades, through its resistant spores (chlamydospores) or as an endophyte on alternative hosts [19]. Organic amendments enriched with beneficial microorganisms have already proven to boost soil suppressiveness and reduce FWB intensity $[20,21]$. Thus, the SPoS could also be explored as an alternative to manage FWB, yet this has not been investigated to date. This study explored the potential of $P$. ostreatus and SPoS to suppress Foc race 1 in vitro and in vivo on potted plants.

\section{Materials and Methods}

This study was conducted between 2016 and 2020 at the National Agricultural Research Laboratories (NARL) of the National Agricultural Research Organization, Kawanda in Uganda. The study comprised in vitro laboratory experiments and pot experiments in a greenhouse.

\subsection{Fungal Strains, Culture Conditions, and Plant Material}

Healthy looking fruiting bodies of P. ostreatus (Supplementary Figure S1) were obtained from MULTIMUSH Limited, a national mushroom research for development company in Uganda. Pure fungal colonies of $P$. ostreatus were obtained by culturing approximately $5 \mathrm{~mm}$ square tissue stubs excised from the fruiting bodies on potato dextrose agar (PDA; Difco Laboratories, Detroit, MI, USA) plates and maintained through regular sub-culturing. The most vigorous isolate of P. ostreatus was used for the subsequent studies.

The SPoS used in this study were obtained from a mushroom incubation facility hosted at NARL, Kawanda. The mushroom gardens were made of cotton seed waste. Fusarium 
oxysporum f. sp. cubense (Foc) was isolated from the rhizome of banana plants of the cv. 'Ndizi' (i.e., 'Sukari ndizi', Silk, AAB) showing typical Fusarium wilt symptoms at NARL, Kawanda $\left(0.40522^{\circ} \mathrm{N}, 032.53283^{\circ} \mathrm{E}, 1182\right.$ m.a.s.l.), in central Uganda. The pathogen was cultured on PDA plates and incubated at $28{ }^{\circ} \mathrm{C}$ in the dark for 7 days and subsequently confirmed as Foc using species-specific PCR primers [22]. A single spore colony of the initial culture was inoculated into five test plants of ' $\mathrm{Ndizi}^{\prime}$ and observed to cause characteristic above - and below — ground Fusarium wilt symptoms and subsequently used in the study.

Tissue culture derived banana plantlets having at least four functional leaves were obtained from a certified banana company_Agro-Genetic Technologies (AGT). Two banana varieties were used: 'Ndizi' which is highly susceptible to Foc [23] and 'Mbwazirume' (Musa AAA), an East African Highland (EAH) banana previously reported as resistant to Foc [24].

\subsection{In Vitro Interactions of Pleurotus ostreatus and Fusarium oxysporum f. sp. cubense}

The in vitro screening assays were performed in $90 \mathrm{~mm}$ Petri plates with PDA. Mycelial plugs of about $3 \mathrm{~mm}$ square were cut out with a sterile blade from actively growing mycelial edge of P. ostreatus and Foc and placed $70 \mathrm{~mm}$ apart on either ends of the same Petri dish. Controls containing only plugs of each fungus were simultaneously set up in separate Petri dishes (Supplementary Figure S2).

Petri dishes were then incubated at $28^{\circ} \mathrm{C}$ and monitored for 30 days. Growth characteristics such as overgrowth, contact inhibition, and distance inhibition of the fungal organisms were visually observed, and growth was measured after every three days. The experiment was repeated four times, with four replicates per treatment in each experiment.

Stubs of $2 \mathrm{~mm}$ were cut out 12 days post inoculation (d.p.i.) from (i) the Foc only control plate, (ii) the Foc-P. ostreatus co-culture interaction zone, and (iii) the Foc end close to the Foc-P. ostreatus co-culture interaction zone, using a $2 \mathrm{~mm}$ Harris Micro-punch (SigmaAldrich, Merck KGaA, Darmstadt, Germany). The stubs were then broken down in $3 \mathrm{~mL}$ of sterile distilled water using a sterile metallic rod and vortexed (Scientific Industries, Inc, Bohemia, NY, USA) to form a fine suspension for enumeration of Foc inoculum. Each of the $2 \mathrm{~mm}$ stubs above acted as a treatment, and a total of 6 stubs were cut per zone. $1 \mathrm{~mL}$ of the original $3 \mathrm{~mL}$ suspension was serially diluted with distilled water to $10^{-4}$. The suspension was vortexed between each dilution step to ensure a homogenous suspension. A volume of $20 \mu \mathrm{L}$ of the suspension of each dilution was put into each side of the hemocytometer groove, and the spores in zones A-E of both sides of the hemocytometer were counted. The above procedure was repeated in triplicates, and the total spore count per replicate was computed as below:

Spores $/ \mathrm{mL}=(n) \times$ the dilution used $/ 0.02$; where $n=$ the average spore count per square.

\subsection{Effect of Extracts from Spent P. ostreatus Substrate (SpoS) on F. oxysporum f. sp. cubense (Foc) Mycelia Growth}

Assessment of the inhibitory effect of SPoS was conducted as described by Szczech [25] and Bernal-Vicente et al. [26]. Different air-dry weights ( $1 \mathrm{~g}, 5,10,15,20,25$, and $50 \mathrm{~g})$ of SPoS were mixed with $100 \mathrm{~mL}$ of double distilled water resulting in $0 \% w / v, 1 \% w / v$ to $50 \% w / v$ of SPoS concentrations. The mixture was vortexed for $10 \mathrm{~min}$ and left to stand at $28^{\circ} \mathrm{C}$ for $48 \mathrm{~h}$, after which it was vortexed again and filtered through sterile cheese cloth. Half of each filtrate concentration was sterilized in an autoclave at $121^{\circ} \mathrm{C}$ and 15 psi of pressure for $15 \mathrm{~min}$ to destroy or denature any biotic components of the filtrate. PDA media prepared with a low water content was filled with the unsterilized and sterilized filtrates above in three replicates. Plates filled with sterile water only served as controls. Thus, there were a total of 15 treatments. Discs $(5 \mathrm{~mm}$ ) of 10-day old Foc cultures on PDA were then placed in the center of each plate and incubated at $28{ }^{\circ} \mathrm{C}$. The plates were observed daily over a period of 30 days. Colony diameters of the Foc mycelia were measured daily from the second day over a period of 12 days. The experiment was repeated twice, with three replicates per treatment in each experiment. 
2.4. Greenhouse Evaluation of Spent P. ostreatus Substrate (SpoS) against Fusarium oxysporum $\mathrm{f}$. sp. cubense

The greenhouse experiment determined the effect of a one-time application of SPoS on FWB severity in potted tissue culture-derived plants. Pot experiments were conducted using pre-sterilized Foc inoculated soils and unsterilized naturally infested soils. Pots of $2.5 \mathrm{~L}$ volume, able to take up to $2.3 \mathrm{~kg}$ of fresh soil at full capacity were used for the experiments.

Presterilized soil inoculations: Loam soil collected from an area with no history of Foc was sterilized for this study. The pot treatments comprised (i) a control treatment in which soil was not inoculated with Foc or SPoS, (ii) soils inoculated with $80 \mathrm{~g}$ of SPoS (i.e., 30\% $v / v$ of the pots) only, (iii) soils inoculated with $50 \mathrm{~g}$ of Foc colonized millet grain only, and (iv) soils inoculated with a combination of $80 \mathrm{~g} \mathrm{SPoS}$ and $50 \mathrm{~g}$ millet grain colonized by Foc. The mean number of Foc colony-forming units (cfu) in $1 \mathrm{~g}$ of Foc colonized millet grain was estimated to be approximately 7.6 million/g using the standard laboratory procedures described above. Effort was made to thoroughly mix soils with either Foc or SPoS to ensure a more homogenous mixture. For trials in which Foc was combined with SPoS, the Foc inoculum was allowed to establish for two weeks before the introduction of SPoS. The SPoS was also allowed to establish for two weeks before introduction of banana plantlets. Thus, the time from Foc inoculation to introduction of banana plantlets was 4 weeks and 2 weeks from addition of SPoS to introduction of banana plantlets. Plantlets of two banana genotypes, a susceptible cultivar 'Ndizi' and a resistant cultivar 'Mbwazirume', were used in this study. Four plants per genotype, each acting as a replicate, were used per treatment, and the experiment was repeated twice.

Naturally infested soils: Naturally infested soils (mean Foc cfu $=0.53 \times 10^{6} \mathrm{~g}^{-1}$ ) obtained from a hotspot within the NARL research station were assessed to mimic field conditions where multiple factors potentially affect interactions between microbial organisms. The experiment comprised only the susceptible 'Ndizi' cultivar planted in pots filled: (i) with Foc infested field soils only, (ii) Foc infested field soils thoroughly mixed with $80 \mathrm{~g}$ of SPoS, (iii) pre-sterilized soil thoroughly mixed with SPoS, and (iv) pre-sterilized soil only. For treatments with P. ostreatus, banana plantlets were introduced two weeks after the addition of SPoS. The rest of the steps were as for the experiment using sterilized soils above. Four plants were used per treatment, each acting as a replicate.

Data collection: The potted plants were regularly watered and monitored for a period of 2.5 months. Data collected from the pot trials included plant girth at soil level and height, the time to first foliar symptom expression, foliar symptom incidence (i.e., number of infected plants), and severity and corm damage score. A modified corm damage score (scale 0 to $5 ; 0=$ no disease and $5=$ severe corm damage) and leaf symptom severity score (scale 0 to $5 ; 0=$ no yellowing of leaves and $5=$ dead plant) developed by Viljoen et al. [27] was used.

\subsection{Data Analysis}

In the in vitro culture studies, the growth of the two fungi were compared on the co-culture plates and individual plates to ascertain suppressive effects from either fungus using analysis of variance (ANOVA) computed using the $\mathrm{R}$ statistical package [28]. The numbers of Foc spores in the different zones of the co-culture plates and Foc control plates were also compared.

The mean values of the diameters of Foc mycelia growth in SPoS extracts (sterilized and unsterilized) and controls of the in vitro studies were also subjected to ANOVA. Mean values of the data collected from the greenhouse pot trials for the different variables were analyzed using ANOVA. Visualizations of data were implemented using the R package 'ggplot2' [29]. 


\section{Results}

3.1. In Vitro Interaction between Pleurotus ostreatus and Fusarium oxysporum f. sp. cubense

Both, Foc and P. ostreatus, showed similar growth patterns on solid PDA media in single cultures (Figure 1A, right panel). However, dual cultures of P. ostreatus-Foc showed strong interactions starting at the 6th d.p.i. when the two fungi came into contact. At that time-point, P. ostreatus hyphae were observed to thicken at the point of contact with Foc mycelia, followed by development of an inhibition zone (Figure 1B, middle panel). After 9 d.p.i., the thickened P. ostreatus mycelia, though at a slower rate compared to the single P. ostreatus control cultures, were observed to grow over and into the hyphae growing zone of Foc. At that time-point Foc hyphae turned brown and seemed to gradually degenerate in the zone of interaction (Figure 1A left panel and Figure 1B middle and right panels).
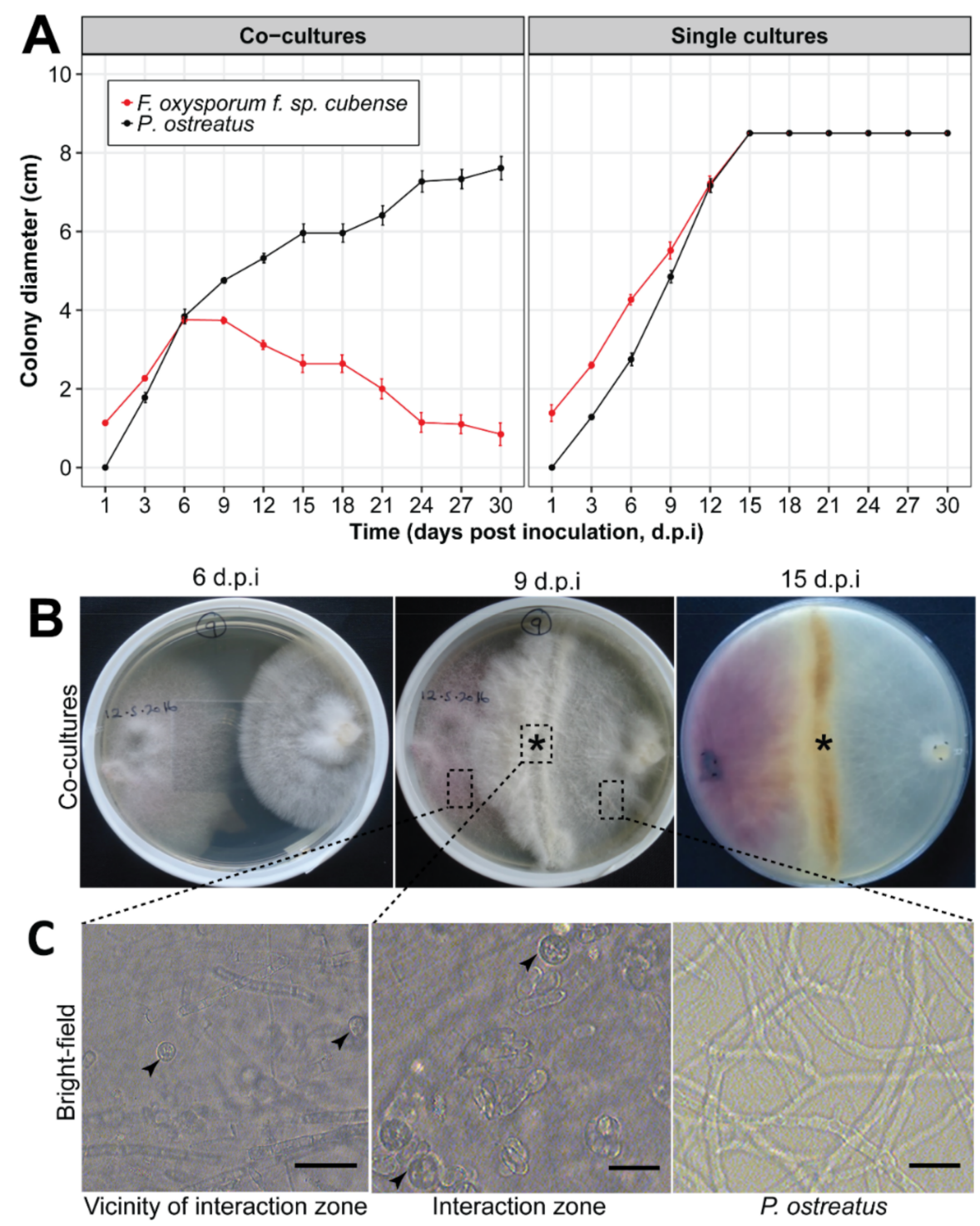

Figure 1. (A) Mycelia growth of co-culture and sole cultures for Fusarium oxysporum $\mathrm{f}$. sp. cubense (Foc) and Pleurotus ostreatus (Po). (B) In vitro Po-Foc co-culture plates at 6-, 9-, and 15-days post inoculation (d.p.i.). (C) Microscopic images $(40 \times)$ from three zones of the Foc-Po co-culture plate showing Foc zone next to the Po-Foc interaction zone, the Po-Foc interaction zone, and the Po zone. The asterisk $\left(^{*}\right)$ show thickened Po mycelia at 9 d.p.i. and subsequent denaturing of Foc mycelia at 15 d.p.i. Black arrows show Foc chlamydospores. Bars represent $1 \mu \mathrm{m}$ magnification. 
At 12 d.p.i. a significantly $(p<0.05)$ lower number of $F o c$ spore counts $\left(11.7 \times 10^{3}\right)$ was obtained in the P. ostreatus-Foc interaction zone when compared to the zone in the vicinity of the point of interaction between P. ostreatus and Foc $\left(68.7 \times 10^{3}\right.$ spores $)$ and the Foc single culture control (117.5 $\times 10^{3}$ spores). Micro and macro-conidia dominated the Foc culture plates. However, samples collected from P. ostreatus-Foc interactions zones and their vicinity (co-cultured plates) were dominated by Foc chlamydospores, with a lower density observed in the P. ostreatus-Foc interaction zone (Figure 1C).

\subsection{Effect of SPoS Extracts on Fusarium oxysporum f. sp. cubense Growth}

Mycelia of Foc growing on PDA amended with autoclaved SPoS filtrate covered entire plates. In contrast, Foc growth was restricted and limited in plates with the unsterilized SPoS filtrate. The mycelia of Foc in these plates turned brown and appeared to have degenerated as for the zone of interaction of the P. ostreatus-Foc co-culture plate above (c.f. Figure 1B).

No differences $(p>0.05)$ in Foc colony growth were observed between PDA amended with sterilized SPoS extracts of varying concentrations of $1 \%, 5 \%, 10 \%, 15 \%, 20 \%, 25 \%$, and $50 \%$ ( $w / v$ of SPoS to sterile water) and the sterile water control (Figure 2 left panel). In contrast, Foc colony growth on PDA supplemented with unsterilized SPoS filtrates (concentrations as above) was significantly $(p<0.05)$ suppressed (Figure 2 right panel). No significant $(p>0.05)$ differences in Foc suppression were however observed between the different concentrations of the unsterilized SPoS filtrate.

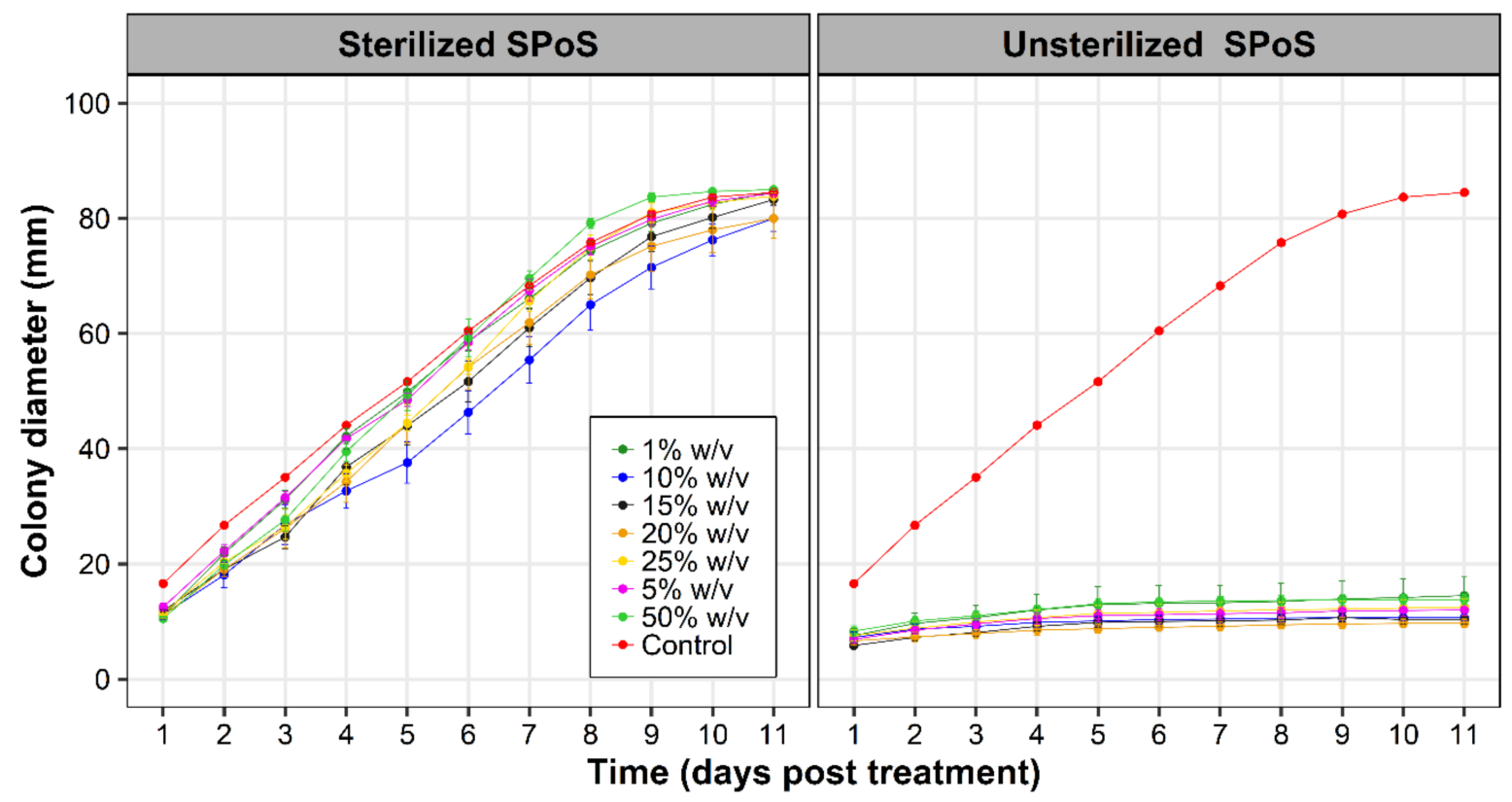

Figure 2. Mycelial growth of Fusarium oxysporum f. sp. cubense on potato dextrose agar (PDA) amended with varying concentrations $(\% w / v)$ of sterilized (left panel) and un-sterilized (right panel) filtrates of spent Pleurotus ostreatus substrate (SPoS).

3.3. Greenhouse Evaluation of Spent P. ostreatus Substrate (SpoS) against Fusarium oxysporum $\mathrm{f}$. sp. cubense

The inoculation process using the Foc R1 isolate was efficient to cause FWB in both banana cultivars and allowed a clear cultivar discrimination regarding resistance levels (Figure 3A,D). In both the susceptible cultivar 'Ndizi' and resistant cultivar 'Mbwazirume', typical external symptoms of FWB were not observed at termination of the experiment, i.e., 2.5 months post inoculation. 

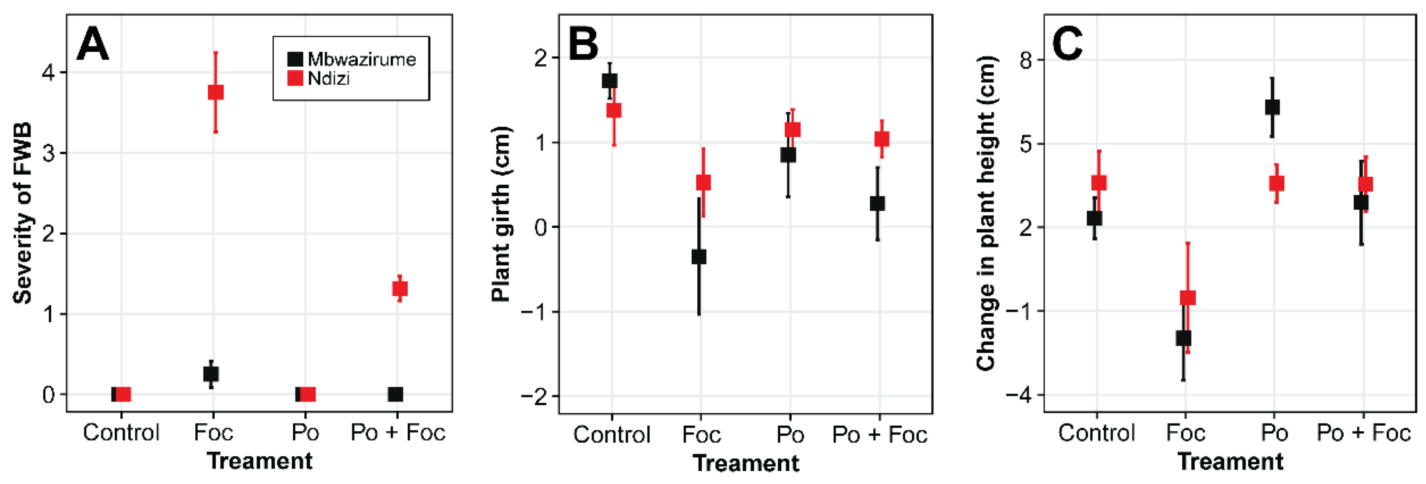

D

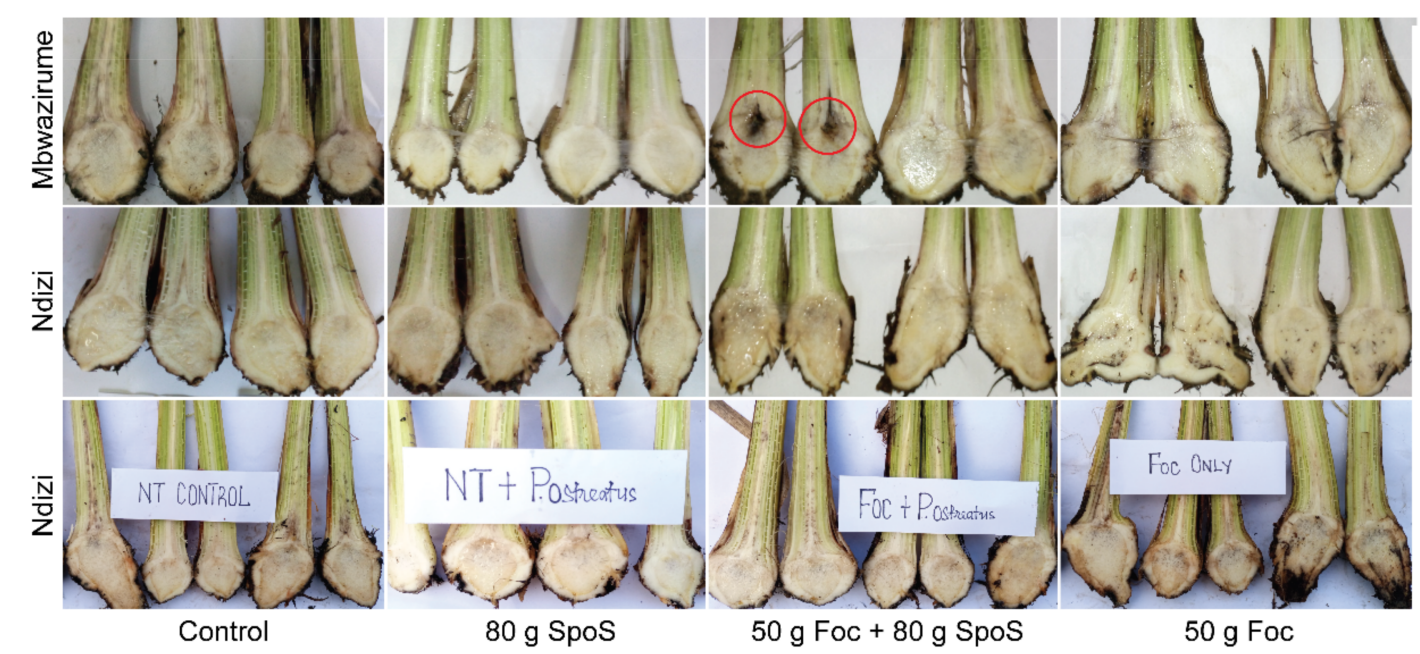

Figure 3. Severity of Fusarium wilt of banana (FWB) in corms (scale: 0 to 5) (A), pseudostem girth (B), plant height (C), and corm tissues appearance (D) of potted banana plants (resistant 'Mbwazirume' (AAA) and susceptible 'Ndizi' (AAB) cultivars) at 2.5 months after being subjected to different treatment combinations. The soils were sterilized and subjected to different treatments that included: a control in which the sterile soil was not amended, soils amended with $80 \mathrm{~g}$ of spent P. ostreatus substrate (SPoS), soils amended with $80 \mathrm{~g} \mathrm{SPoS}$ and $50 \mathrm{~g}$ Foc colonized millet grain, and soils amended only with $50 \mathrm{~g}$ of Foc colonized grain. The zones circled in red show damage due to banana weevils.

When the effect of SPoS was analyzed, it was observed that a reduction on FWB severity occurred in both cultivars. In the susceptible cultivar, a significantly $(p<0.05)$ lower corm discoloration was observed in Foc inoculated soils amended with SPoS (score $=1.31$ ) compared with the un-amended Foc inoculated soils (3.75) (Figure 3A,D). While severe corm discoloration was observed in 'Ndizi', only slight corm discoloration was observed in the resistant cultivar 'Mbwazirume'. In the resistant cultivar, no corm discoloration was observed in the SPoS amended soil whereas a low (0.25) corm discoloration occurred in the Foc only soils (Figure 3A,D). However, corm discoloration in the Foc only soils for the resistant cultivar was not significantly different $(p>0.05)$ from that of the Foc inoculated soils amended with SPoS. For both the resistant and susceptible cultivar, no corm discoloration occurred in the control treatments (i.e., un-inoculated soils and soils amended with SPoS only).

The plants in Foc inoculated pots also had a retarded growth (Figure 3B,C), while $P$. ostreatus increased plant height and girth, even where both Foc and P. ostreatus had been jointly applied. No significant $(p>0.05)$ interactions were observed between the treatments and cultivars despite a higher increment in plant height and pseudostem girth in the control and P. ostreatus inoculated plants. Lower plant height and girth values were observed for 'Mbwazirume' plants treated with Foc despite resisting the pathogen (Figure 3B,C). 


\subsection{Suppression of Foc in 'Ndizi' Plants by SPoS in Naturally Foc-Infested Soils}

Similar trends in corm damage to that in the artificially inoculated soils were observed in the 'Ndizi' plants planted in the naturally infected soils. Foc corm damage or discoloration was observed in both the un-amended naturally Foc-infested soils and the naturally Foc-infested soils amended with SPoS. However, SPoS significantly reduced the corm discoloration score (0.88) due to Foc when compared to 2.75 in the un-amended naturally Focinfested treatment (Table 1). No corm damage occurred in plants treated with P. ostreatus only and the control treatments that had sterile soil.

Table 1. Corm damage score (scale from 0 to $5 ; 0=$ no damage, $5=$ very severe damage) and change in pseudostem girth, height, and dry root weight of 'Ndizi' plants in (i) soils naturally infested with Fusarium oxysporum f. sp. cubense Race 1 (Foc), (ii) Foc infested soils amended with $80 \mathrm{~g}$ of spent P. ostreatus substrate (SPoS), (iii) sterile soils amended with SPoS, and (iv) an unamended sterile soil as control.

\begin{tabular}{ccccc}
\hline Treatments & $\begin{array}{c}\text { Corm Damage Score } \\
( \pm \mathbf{s d})\end{array}$ & $\begin{array}{c}\text { Plant Girth } \\
(\mathbf{c m} \pm \mathbf{s d})\end{array}$ & $\begin{array}{c}\text { Plant Height } \\
(\mathbf{c m} \pm \mathbf{s d})\end{array}$ & $\begin{array}{c}\text { Dry Root Weight } \\
(\mathbf{g} \pm \mathbf{s d})\end{array}$ \\
\hline Foc-infested soil & $2.75 \pm 0.50$ & $-0.40 \pm 0.34$ & $-0.90 \pm 0.52$ & $5.20 \pm 1.41$ \\
Foc-infested soil + SPoS & $0.88 \pm 0.83$ & $0.43 \pm 0.69$ & $0.74 \pm 0.48$ & $3.79 \pm 1.30$ \\
Sterile soil + SPoS & $0.00 \pm 0.00$ & $1.08 \pm 1.42$ & $2.38 \pm 1.55$ & $3.02 \pm 1.81$ \\
Control (sterile soil) & $0.00 \pm 0.00$ & $1.60 \pm 1.80$ & $2.28 \pm 2.14$ & $4.53 \pm 1.55$ \\
$p$ value & $1.53 \mathrm{e}-05$ & 0.1718 & 0.0040 & 0.2714 \\
\hline
\end{tabular}

In general, a decline in the aboveground growth of plants as shown by the changes in plant girth and height in Foc infected soils was observed, while only a retardation of growth occurred in the P. ostreatus treated plants in naturally infested soils (Table 1). Overall, no significant difference was observed between the treatments for plant girth, despite the decline in girth of plants in Foc infested soils. A significant difference $(p=0.004)$ in plant height was observed, with higher values visible for the sterile $P$. ostreatus amended soils and the control (Table 1). In contrast, dry root weight was higher in the Foc infested soil followed by the control and the least in the sterile soil with P. ostreatus, though no significant differences $(p>0.05)$ occurred between the treatments (Table 1).

\section{Discussion}

In this work, we show evidence, for the first time, that $P$. ostreatus can suppress Foc race 1 both in vitro and in greenhouse experiments. During the in vitro interactions P. ostreatus hyphae were observed to enlarge and become more vigorous at points of contact with Foc hyphae, suggesting that putative competition mechanisms are involved. The enlargement and increased vigor of P. ostreatus hyphae was subsequently followed by the degeneration of Foc on media, suggesting that, in addition to competition, antibiosis, and predation mechanisms could also be involved in Foc suppression by P. ostreatus. These interactions could also explain the lower number of Foc chlamydospores observed in the zone in which Foc degenerated due to interaction with P. ostreatus, relative to that in the zone adjacent to the point/zone of interaction. The higher number of Foc chlamydospores in this adjacent zone could be related to defense and survival mechanisms of Foc to overcome P. ostreatus. For example, in the interaction between phytobacterium Ralstonia solanacearum and the fungi Fusarium fujikuroi (causal agent of foolish seedling disease of rice), the bacterium has been reported to produce a lipopeptide (ralsolamycin) that triggers production of chlamydospores in the fungi [30].

Antifungal activity, from unsterilized substrates of $P$. ostreatus and other mushroom species, has been reported against other formae specialis of $F$. oxysporum, such as F. o. f. sp lycopersici in tomatoes [15], F.o. f. sp. melonis in melon crop [31], and F. of. sp. cepae, which causes basal rot disease in shallot pots [32]. Our results also showed that the unsterilized filtrate of SPoS significantly inhibited the growth of Foc, even at low concentration $(1 \% w / v)$.

The fact that the sterilized filtrate had no effect on the growth of Foc mycelia suggests that the suppressive effect of SPoS could be attributed to heat labile secondary metabolites, 
from P. ostreatus and inherent micro-organisms in the SPoS. P. ostreatus compounds, such as Pleurostrin, have been shown to have antifungal activity against Fusarium oxysporum, Mycosphaerella arachidicola and Physalospora piricola [33-35]. The substrate of P. ostreatus has also been reported to contain a wide diversity of microorganisms with strong antagonism toward pathogenic fungi [15,31]. Adedeji and Aduramigba [15] recovered Trichoderma viridae, fluorescent Pseudomonas spp., Baccilus spp., Aspergillus terrus and Penicillum spp., from spent substrate of P. ostreatus that had a strong antagonism to Fol [15]. Spent mushroom substrate teas have also been shown to suppress the phytopathogens Phytophthora capsici and Phytophthora parasitica, in vitro and in vivo [14]. Metabolomic studies and the purification of specific compounds from SPoS involved on Foc suppression, as well as mechanisms involved need further investigation.

In potted trials, suppression of Foc, shown by a lower corm damage in potted plants treated with P. ostreatus, was observed in both pre-sterilized artificially inoculated soils and naturally infested unsterilized soils. Failure of bio-control agents under natural field conditions due to the interactions with environmental and bio-physical factors has been a major constraint to their deployment. The success of $P$. ostreatus in naturally infested unsterilized soils suggests that $P$. ostreatus has the potential to succeed under field conditions. However, further studies to determine the effect of P. ostreatus on Foc under field conditions and different levels of management are still needed, for more concrete recommendations.

As expected, 'Ndizi' the Foc susceptible cultivar recorded a higher level of Foc damage. The resistant cultivar 'Mbwazirume' also registered some corm damage, albeit low. This cultivar and other EAH types (Musa AAA) have been reported to be immune or to resist Foc race 1 [24].

P. ostreatus spent substrate also increased overall aboveground biomass of the plants. Marin et al. [14] also reported spent mushroom substrate teas to promote plant growth. Increased plant vigor could have also improved the plants' ability to resist Foc infection. Lower plant height and girth scores for the resistant 'Mbwazirume' cultivar can be attributed to banana weevil (Cosmopolites sordidus) damage that was observed in some of the assessed corm tissues. 'Mbwazirume' like other EAH banana cultivars is highly susceptible to banana weevils [36].

The root weight of plants was increased by Foc infection. This could be due to the formation of more roots to replace those lost or damaged by the infection. In beans, development of lateral roots above the initial sites of infection caused by F. solani f. sp. phaseoli infection and other root rot pathogens, such as Pythium spp. and Rhizoctonia solani, has been reported [37].

\section{Conclusions}

This study shows a high potential of the P. ostreatus spent substrate as part of the management practices to reduce the impact of FWB. Given the current increase in mushroom production in East Africa, its application could ensure an effective recycling of crop wastes and higher crop productivity, while reducing the burden of FWB. The fact that $P$. ostreatus will contribute to the incomes and nutrition of the farming communities makes its use a relatively cheap, environmentally sound, and farmer friendly option for sustainable management of FWB. Additional field studies under different disease pressure and environmental and management settings to evaluate the efficacy of $P$. ostreatus are recommended and ongoing. In addition, studies to elucidate the inhibition mechanisms of $P$. ostreatus (compounds), P. ostreatus spent substrate, and associated micro-organisms are ongoing. Studies on the efficacy of different $P$. ostreatus substrate types are also recommended.

Supplementary Materials: The following are available online at https: / www.mdpi.com/article/ 10.3390/jof7110946/s1, Figure S1: Pleurotus ostreatus garden showing healthy fruiting bodies, Figure S2: Overview of experimental setup to determine the in vitro interspecific interaction, antagonistic effect and mycoparasitism of Pleurotus ostreatus (Po) on Fusarium oxysporum f. sp. cubense (Foc). 
Author Contributions: Conceptualization, W.O. and E.W.; data curation, W.O.; formal analysis, W.O. and E.W.; investigation, W.O., E.W. and A.F.T.; methodology, W.O., E.W., A.F.T., M.D., S.-J.Z. and G.B.; resources, G.B.; visualization, W.O. and E.W.; writing-original draft, W.O. and A.F.T.; writing-review and editing, W.O., E.W., A.F.T., M.D., S.-J.Z. and G.B. All authors have read and agreed to the published version of the manuscript.

Funding: This study was supported by funds from the CGIAR Fund Donors (http:/ / www.cgiar.org/ about-us/our-funders / (accessed on 10 August 2021)) through the CGIAR Research Program on Roots, Tubers and Bananas.

Data Availability Statement: The raw data supporting the conclusions of this manuscript will be made available by the authors, without undue reservation, to any qualified researcher.

Acknowledgments: We are grateful for the support of the CGIAR Research Program on Roots, Tubers and Bananas. We also acknowledge Dorcus Nassazi and Leniru Salome for their assistance with laboratory work. We acknowledge MULTIMUSH Limited for providing the spent P. ostreatus substrate gardens.

Conflicts of Interest: The authors declare that the research was conducted in the absence of any commercial or financial relationships that could be construed as or result in a potential conflict of interest.

\section{References}

1. Sánchez, C. Cultivation of Pleurotus ostreatus and other edible mushrooms. Appl. Microbiol. Biotechnol. 2010, 85, 1321-1337. [CrossRef]

2. de Carvalho, C.S.M.; Sales-Campos, C.; de Andrade, M.C.N. Mushrooms of the Pleurotus genus: A review of cultivation techniques. Interciencia 2010, 35, 177-182.

3. Fernández-Fueyo, E.; Ruiz-Dueñas, F.J.; López-Lucendo, M.F.; Pérez-Boada, M.; Rencoret, J.; Gutiérrez, A.; Martínez, A.T. A secretomic view of woody and nonwoody lignocellulose degradation by Pleurotus ostreatus. Biotechnol. Biofuels 2016, 9, 49. [CrossRef] [PubMed]

4. Williams, B.C.; McMullan, J.T.; McCahey, S. An initial assessment of spent mushroom compost as a potential energy feedstock. Bioresour. Techhnol. 2001, 79, 227-230. [CrossRef]

5. Prabu, M.; Jeyanthi, C.; Kumuthakalavalli, R. Spent mushroom substrate: An enriched organic manure for improving the yield of Vigna unguiculata (L.) Walp (Cowpea) leguminous crop. Scrut. Int. Res. J. Agric. Plant Biotechnol. Bioprod. 2014, 1, 7-14.

6. Jasińska, A. Spent mushroom compost (SMC)-retrieved added value product closing loop in agricultural production. Acta Agrar. Debr. 2018, 150, 185-202. [CrossRef]

7. Paszczynski, A.; Crawford, R. Recent advances in the use of fungi in environmental remediation and biotechnology. Soil. Biochem. 2000, 10, 379-422.

8. Pointing, S. Feasibility of bioremediation by white-rot fungi. Appl. Microbiol. Biotechnol. 2001, 57, $20-33$.

9. Velázquez-Cedeño, M.; Farnet, A.M.; Billette, C.; Mata, G.; Savoie, J.M. Interspecifc interactions with Trichoderma longibrachiatum induce Pleurotus ostreatus defence reactions based on the production of laccase isozymes. Biotechnol. Lett. 2007, 29, 1583-1590. [CrossRef] [PubMed]

10. Baldrian, P. Increase of laccase activity during interspecific interactions of white-rot fungi. FEMS Microbiol. Ecol. 2004, 50, 245-253. [CrossRef]

11. Alves, M.J.; Ferreira, I.C.; Dias, J.F.; Teixeira, V.; Martins, A.; Pintado, M. A review on antimicrobial activity of mushroom (Basidiomycetes) extracts and isolated compounds. Planta Med. 2012, 78, 1707-1718. [CrossRef] [PubMed]

12. Rayner, A.D.M.; Griffith, G.S.; Wildman, H.G. Induction of metabolic and morphogenetic changes during mycelia interactions among species of higher fungi. Biochem. Soc. Trans. 1994, 22, 389-394. [CrossRef]

13. Phan, C.W.; Sabaratnam, V. Potential uses of spent mushroom substrate and its associated lignocellulosic enzymes. Appl. Microbiol. Biotechnol. 2012, 96, 863-873. [CrossRef] [PubMed]

14. Marin, F.; Dianez, F.; Santos, M.; Carretero, F.; Gea, F.J.; Castaneda, C.; Navarro, M.J.; Yau, J.A. Control of Phytophthora capsici and Phytophthora parasitica on pepper (Capsicum annuum L.) with compost teas from different sources, and their effects on plant growth promotion. Phytopathol. Mediterr. 2014, 53, 216-228.

15. Adedeji, K.O.; Aduramigba, M.A.O. In vitro evaluation of spent mushroom compost on growth of Fusarium oxysporium f. sp. lycopersici. Adv. Plants Agric. Res. 2016, 4, 332-339.

16. Viljoen, A. The status of Fusarium wilt (Panama disease) of banana in South Africa. S. Afr. J. Sci. 2002, 98, 341-344.

17. Ploetz, R.C. Fusarum wilt of banana. Phytopathology 2015, 105, 1512-1521. [CrossRef]

18. Dita, M.; Teixeira, L.A.J.; O’Neill, W.; Pattison, A.B.; Weinert, M.P.; Li, C.Y.; Zheng, S.J.; Staver, C.; Thangavelu, R.; Viljoen, A. Current state of Fusarium wilt of banana in the subtropics. Acta Hort. 1272, 2020, 45-56. [CrossRef]

19. State of Queensland. Guide to Alternative Hosts of Panama Disease in Australia; Hort Innovation Queensland: Queensland, Australia, 2020; p. 47. 
20. Zhang, N.; Xin, H.E.; Zhang, J.; Raza, W.; Yang, X.-M.; Ruan, Y.-Z.; Shen, Q.-R.; Huang, Q.-W. Suppression of Fusarium wilt of banana with application of bio-organic fertilizers. Pedosphere 2014, 24, 613-624. [CrossRef]

21. Akyuz, M.; Kirbag, S. Antimicrobial activity of Pleurotus eryngii var. ferulae grown on various agro-wastes. Eur. Asian, J. BioSci. 2009, 3, 58-63. [CrossRef]

22. Mishra, P.K.; Fox, R.T.; Culham, A. Development of a PCR-based assay for rapid and reliable identification of pathogenic Fusaria. FEMS Microbiol. Lett. 2003, 218, 329-332. [CrossRef] [PubMed]

23. Pegg, K.G.; Moore, N.Y.; Sorensen, S. Fusarium wilt in the Asian Pacific region. In Proceedings of the International Symposium on Recent Development in Banana Cultivation Technology, Pingtung, Taiwan, 14-18 December 1992; Valmayor, R.V., Hwang, S.C., Ploetz, R.C., Lee, S.W., Roa, V.N., Eds.; Taiwan Banana Research Institute: Pingtung, Taiwan, 1993; pp. $255-314$.

24. Molina, A.B.; Sinohin, V.O.; Fabregar, E.G.; Ramillete, E.B.; Yi, G.; Sheng, O.; Viljoen, A. Resistance to Fusarium oxysporum f. sp. cubense tropical race 4 in African bananas. In Proceedings of the XXIX International Horticultural Congress on Horticulture: Sustaining Lives, Livelihoods and Landscapes (IHC2014): IX 1114, Brisbane, Australia, 17-22 August 2014; pp. 107-110.

25. Szczech, M.M. Suppressiveness of vermicompost against Fusarium wilt of tomato. J. Phytopathol. 1999, 147, 155-161. [CrossRef]

26. Bernal-Vicente, A.; Ros, M.; Tittarelli, F.; Intrigliolo, F.; Pascual, J.A. Citrus compost and its water extract for cultivation of melon plants in greenhouse nurseries. Evaluation of nutri-active and biocontrol effects. Bioresour. Technol. 2008, 99, 8722-8728. [CrossRef]

27. Viljoen, A.; Mahuku, G.; Massawe, C.; Ssali, R.T.; Kimunye, J.; Mostert, G.; Ndayihanzamaso, P.; Coyne, D.L. Banana Pests and Diseases: Field Guide for Disease Diagnostics and Data Collection; International Institute of Tropical Agriculture (IITA): Ibadan, Nigeria, 2016.

28. R Core Team. R: A Language and Environment for Statistical Computing; R Foundation for Statistical Computing: Vienna, Austria, 2018. Available online: https:/ / www.R-project.org/ (accessed on 19 July 2021).

29. Wickham, H. Elegant Graphics for Data Analysis; Springer: Berlin/Heidelberg, Germany, 2009; p. 35.

30. Spraker, J.E.; Sanchez, L.M.; Lowe, T.M.; Dorrestein, P.C.; Keller, N.P. Ralstonia solanacearum lipopeptide induces chlamydospore development in fungi and facilitates bacterial entry into fungal tissues. Int. Soc. Microbial. Ecol. J. 2016, 10, 2317-2330. [CrossRef]

31. Suárez-Estrella, F.; Bustamante, M.A.; Moral, R.; Vargas-García, M.C.; López, M.J.; Moreno, J. In vitro control of Fusarium wilt using Agro-industrial sub-product-based composts. J. Plant Pathol. 2012, 94, 59-70.

32. Yusidah, I.; Istifadah, N. The abilities of spent mushroom substrate to suppress basal rot disease (Fusarium oxysporum f. sp. cepae) in shallot. Int. J. Biosci. 2018, 13, 440-448.

33. Owaid, M.N.; AL Saeedi, S.S.S.; Abed, I.A.; Shahbazi, P.; Sabaratnam, V. Antifungal activities of some Pleurotus species (Higher Basidiomycetes). Walailak J. Sci. Technol. 2017, 14, 215-224.

34. Chu, K.T.; Xia, L.; Ng, T.B. Pleurostrin, an antifungal peptide from the oyster mushroom. Peptides 2005, 26, 2098-2103. [CrossRef]

35. Rosa, L.H.; Machado, K.M.G.; Jacob, C.C.; Capelari, M.; Rosa, C.A.; Zani, C.L. Screening of Brazilian Basidiomycetes for antimicrobial activity. Mem. Inst. Oswaldo Cruz, Rio de Janeiro 2003, 98, 967-974. [CrossRef]

36. Gold, C.S.; Kiggundu, A.; Abera, R.; Ssendege, A.M.K.; Karamura, E.B.; Bagamba, F.; Wejuli, M.; Karamura, D.; Kalyebara, R. Geographic shifts in highland banana production in Uganda. Acta Hort. 2000, 540, 55-62. [CrossRef]

37. Schwartz, H.F.; Root Rots of Dry Beans. Fact Sheet No. 2.938. Crop Series: Diseases. Colorado State University Cooperative Extension Service. 2021. Available online: https://extension.colostate.edu/docs/pubs/crops/02938.pdf (accessed on 8 November 2021). 